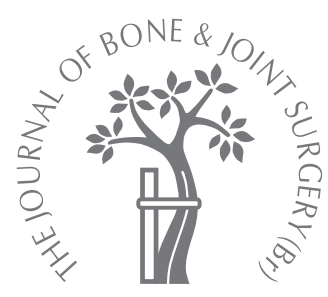

\title{
Transmission of methicillin-resistant Staphylococcus aureus among hospital staff in a German trauma centre
}

\author{
A PROBLEM WITHOUT A CURRENT SOLUTION?
}
A. Kaminski, J. Kammler, M. Wick, G. Muhr, F. Kutscha-Lissberg

From University Hospital

Bergmannsheil, Bochum, Germany

Between October 2001 and February 2002, 324 healthcare workers were screened for methicillin-resistant Staphylococcus aureus (MRSA) by nose and throat swabs. A positive finding led to activation of a standardised control programme for the affected person who was immediately excluded from work. Family members of those who were MRSA-positive were offered screening free of charge. An eradication programme was carried out in the permanent carriers. MRSA was found in 17 (5.3\%) healthcare workers, 11 of whom proved to be permanent carriers, and six temporarily colonised. Three children of a positive healthcare worker showed nasopharyngeal MRSA, the acquisition of which occurred within the hospital. The standardised eradication programme for carriers was successful in most cases but failed in two individuals, whereupon systemic antibiotics were used successfully. The decolonised carriers, observed for more than one year, remained MRSA negative.

Isolation precautions in hospitals do not always prevent hospital staff and their families from acquiring MRSA. The identification of affected employees is difficult because in most cases only asymptomatic colonisation occurs. Screening and eradication can be complicated and costly, and for the affected employees the occupational consequences can be far-reaching as they have no guaranteed legal protection.

Strains of methicillin-resistant Staphylococcus aureus (MRSA) are responsible for almost half of hospital-acquired staphylococcal infections and are associated with increased mortality. ${ }^{1-3}$ In Japan, up to $90 \%$ of Staph. aureus isolated from inpatients in general hospitals are methicillin resistant. ${ }^{4}$ In Europe, Germany has the highest increase in the prevalence of MRSA. ${ }^{1}$ Treatment options are limited, and the expense of MRSA infection is significant. In the USA, the cost of catheter-associated septicaemia caused by MRSA, significantly exceeds that of disease caused by methicillin-sensitive strains. ${ }^{5}$ In Germany, the avoidable costs on a surgical ward are estimated at $€ 9621$ per case of MRSA. ${ }^{6}$

There are several ways in which transmission of MRSA can occur, the most relevant being through contamination of the hands. ${ }^{7}$ Carriers of MRSA, whether patients or healthcare workers, are colonised predominantly in the nares. ${ }^{8}$ This reservoir can cause hand contamination, the strain of which is typically of the nares. ${ }^{9}$ Colonised healthcare workers are generally asymptomatic, but they create a potential reservoir of infection for susceptible patients. They can also transmit the bacteria at home. ${ }^{10,11}$ Symptomatic MRSA infections among healthcare workers have been described. ${ }^{12-14}$ Yet whilst contact isolation and the eradication of MRSA have been noted as important measures in colonised patients, the procedure for colonised healthcare workers remains unclear. Screening them for MRSA, for example, is controversial. Whereas the Dutch recommend screening amongst healthcare workers after exposure to MRSA-positive patients, ${ }^{15}$ the guidelines of the German ${ }^{16}$ and North American specialist associations ${ }^{17}$ are restrictive. Opponents of universal staff screening argue that there is no proof of its benefit, and that there is a risk of stigmatisation of those affected, as well as of exposure to potentially toxic decolonisation procedures. ${ }^{18}$ Therefore selective screening is advised. ${ }^{19}$

The percentage of MRSA carriers among the patients in our surgical department ranged between $3.2 \%$ in 2001 and $5.6 \%$ in 2002. A screening programme was undertaken in healthcare workers in order to control the possible transmission of MRSA from staff to patients. The possibility of MRSA transmission from these workers to their families was also investigated. 
Table I. Standardised methicillin-resistant Staphyloccus aureus eradication regimen. Treatment over five days represents one cycle

\begin{tabular}{ll}
\hline Site & Treatment \\
\hline Nose & Mupirocin nasal ointment (Turixin, SmithKline Beecham, Germany) \\
Throat & $\begin{array}{l}\text { See nose. } \\
\text { In addition, throat irrigation with octenidine dihydrochloride solution } \\
\text { (Octenisept, Schuelke \& Mayr, Norderstedt, Germany) diluted with distilled } \\
\text { water } 1: 4\end{array}$ \\
Skin & $\begin{array}{l}\text { Whole-body washing and hair washing with octenidine dihydrochloride } \\
\text { solution (Octenisept) dilution with tap water } 1: 1\end{array}$ \\
$\begin{array}{l}\text { Skin lesions and nasopharyngeal } 5 \\
\text { lesions }\end{array}$ & No standardised measures but individual treatment by a specialist physician in the relevant discipline 5 days
\end{tabular}

\section{Materials and Methods}

We conducted the study in a level I trauma centre at a 750bed German hospital, where approximately 13000 surgical inpatients are treated annually. Patients with MRSA colonisation or infection are treated according to the contact isolation principle. The affected patient is accommodated in a private room with its own sanitary facilities and only ever leaves this room for diagnostic or therapeutic reasons. All those who enter the room are instructed to wear gloves, masks and gowns. On a daily basis, all wipeable surfaces are disinfected and the bedlinen is changed. Between October 2001 and February 2002, 324 doctors and nurses involved in the treatment of MRSA-positive patients were screened by swabbing the nose and throat. The anterior nares was sampled by rotating a moistened, sterile, cotton-tipped swab in one nostril. The throat was swabbed in a circular motion.

The swabs were cultured on sheep blood agar plates with $4 \% \mathrm{NaCI}$ after inoculation on to approximately one-third of the plate. The inoculum was streaked over the remaining plate in at least two separate thirds. The plates were incubated for 48 hours at $36^{\circ} \mathrm{C}$. Swab cultures were enriched in trypticase soya broth with $10 \% \mathrm{NaCI}$ for 48 hours at $36^{\circ} \mathrm{C}$ and subcultured on to sheep blood agar plates. Identification of Staph. aureus was by morphology, production of DNAse (Oxoid, Basingstoke, United Kingdom) and mannitol (Oxoid) and the presence of free-plasma coagulase using rabbit plasma (bioMérieux, Marcy l'Etoile, France). Oxacillin susceptibility was tested using Mueller-Hinto agar containing $6 \mathrm{mg} / \mathrm{l}$ oxacillin and $4 \% \mathrm{NaCI}$ (Becton Dickinson, Heidelberg, Germany). Isolates were spot inoculated on to agar and incubated for 48 hours at $36^{\circ} \mathrm{C}$ with Staph. aureus ATCC 6538 and 33592 as control strains. All MRSA isolates underwent phage typing to determine possible acquisition routes. Phage typing of the MRSA-colonised patients preceded staff screening.

Detection of MRSA activated a control programme for the affected healthcare worker, whose demographic details, history of MRSA exposure and risk factors, such as skin, or nasopharyngeal lesions, were recorded. The healthcare worker was immediately excluded from work. This was followed by further screening of the nares, throat and perineum and a distinction was made between temporarily colonised persons and permanent carriers. If MRSA was found only once during primary screening and not on three different occasions thereafter, persons were regarded as temporarily colonised. When MRSA was also found on follow-up screening, persons were regarded as permanent carriers. Temporarily-colonised healthcare workers were able to resume work. Permanent carriers were referred to the appropriate medical specialist if the aforementioned risk factors were present, and from there, underwent an eradication programme (Table I) established by the hospital infection control committee for staff and patients alike, according to national recommendations. ${ }^{16}$ At a minimum of two days after a treatment cycle, swabs were repeated from the nose, throat and perineum. If no further MRSA was detected, two further tests were performed on two succeeding days. If these were also negative, the person was regarded as decolonised. If not, a second or third cycle was carried out (Table I), again followed by three tests. Family members of the MRSA-positive worker were offered screening free of charge.

The success of the eradication procedures in the permanent carriers was evaluated by follow-up screening in 2003. Statistical analysis was performed using the KruskalWallis test, with a significance level of $\mathrm{p}<0.05$. The value characteristics (normal distribution) were determined by the Kolmogorov-Smirnov test.

\section{Results}

Methicillin-sensitive Staph. aureus was found in 118 (36.4\%) healthcare workers and MRSA in 17 (5.3\%). According to follow-up screening $11(3.4 \%)$ were permanent carriers and six $(1.8 \%)$ were temporarily colonised. The occupational groups did not differ significantly with regard to the colonisation rate $(\mathrm{p}=0.356$, Kruskal-Wallis test). The family of one MRSA-positive healthcare worker was included. No MRSA was found in the spouse, but their three children had nasopharyngeal colonisation with MRSA. They had no risk factors nor any signs of illness. The carriers among healthcare workers also had no disease attributable to MRSA. Two MRSA-positive healthcare workers had a mild non-febrile upper respiratory tract 
infection when screened. Both were referred to specialists, who excluded lower respiratory tract and nasopharyngeal infection. As there was rapid (less than five days) and complete regression of symptoms, no other microbiological tests were performed. No healthcare worker who tested positive for MRSA had skin lesions.

The acquisition of MRSA occurred within the hospital in all carriers, and the strains found in staff were identical to those in patients.

All MRSA carriers underwent the eradication programme. Nine of 11 healthcare workers and the three children were successfully decolonised after an average of 1.5 treatment cycles. In two healthcare workers the programme failed and even after the third cycle, pharyngeal MRSA was found in both. They were referred to an Ear, Nose, and Throat specialist and decolonisation with systemic antibiotics was instituted. Both received oral treatment for ten days with $800 \mathrm{mg}$ co-trimoxazole (twice daily) and $600 \mathrm{mg}$ rifampicin (once daily). The first follow-up screening was performed two days afterwards and MRSA was not detected on this, or two further tests.

The decolonised carriers, observed for more than one year, remained MRSA negative. One of them left because of a career change and was not available for follow-up.

\section{Discussion}

The percentage of permanent MRSA carriers (3.4\%) among staff in our investigation corresponds to that of MRSA-positive surgical patients. This compares with previously reported rates among staff of between $0.9 \%$ and $13.2 \% .{ }^{3,14}$ Despite agreement that healthcare workers can be involved in spreading MRSA, control measures to prevent this are controversial. ${ }^{8,10,18,19}$ The Robert Koch Institute approves staff screening only when there is increased detection of MRSA in several patients and proven clonal identity. ${ }^{16}$ Blok et $\mathrm{al}^{8}$ argue that isolation precautions do not always prevent healthcare workers from acquiring MRSA, but that post-exposure screening and exclusion of MRSA-positive workers from work are highly effective in protecting other patients. Conversely, Bowler ${ }^{18}$ postulates that there is little evidence that such activity contributes to control. In German-speaking countries, active post-exposure screening of healthcare workers is not routine, primarily because of the consequences for management and hospital staff. In the case of permanent carriers, further employment of that healthcare worker is not advised where there is patient contact without eradication of the bacteria. Continuing to work with uninterrupted use of masks, gown and gloves leads to stigmatisation and cannot be expected of patients or the affected healthcare worker. Decontamination with mupirocin ointment and octenidine dihydrochloride baths proves effective in this and in other investigations, ${ }^{20}$ but does not provide a universal solution. Failure of this regimen can have various causes, from risk factors in the skin or pharynx, such as chronic eczema or tonsillitis, to recurrent colonisation from the contaminated home environment. Allen et $\mathrm{al}^{10}$ describe the case of a nurse in whom permanent eradication of MRSA was not achieved with mupirocin ointment, chlorhexidine washing and systemic antibiotic treatment. Only by thorough cleaning of the contaminated home environment was permanent eradication achieved after ten months, and the authors conclude that early detection and prompt treatment of MRSA in healthcare workers can minimise the risk of home contamination. ${ }^{10}$

The duration of MRSA colonisation is possibly an important parameter in dissemination. However, in our study the home environment was not investigated. If three cycles of the eradication regimen failed, systemic antibiotic treatment was given, which is also reported by others. ${ }^{8,10,14}$ Criticism of systemic antibiotic treatment in asymptomatic persons is unjustified because we found it led to permanent eradication of the organism and resumption of professional work. Risk factors for permanent colonisation must be recognised, and affected healthcare workers referred to appropriate medical specialists. The involvement of healthcare workers with risk factors in the treatment of MRSApositive patients should also be restricted.

Temporary exclusion from work is justifiable and practicable, but expensive. Permanent exclusion was not necessary in our study but it is described in The Netherlands, ${ }^{8}$ with the possible consequence of a career change. Such a drastic professional, social and economic outcome raises the question of whether those affected should be regarded as victims or culprits, and whether colonisation with a multi-resistant organism without manifest disease justifies exclusion from work. Moral support and counselling must be provided, but these do not solve the problem of the risk to the livelihood of those affected and to their personal professional development.

Addressing these problems in legislation is vital. German statutory accident insurance (gesetzliche Unfallversicherung) does consider permanent contamination of healthcare workers with MRSA. Specialist associations, responsible for competence, have been asked for prospective solutions, and approval or rejection of staff screening is not decisive. It is more important to draw up clearly structured policies for colonised staff to include prevention and management of outbreaks among medical staff.

There is one instance in our study in which family screening was undertaken. Most family members were positive MRSA carriers and successfully decontaminated. Other authors have described transmission of MRSA to family members, ${ }^{10,21}$ with Eveillard et al $^{21}$ reporting such transmission in four out of ten families. This demonstrates that transmission of MRSA beyond the healthcare setting may be a source of MRSA colonisation in the community. In their study, Calfee et $\mathrm{al}^{22}$ defined a group of individuals as close contacts of colonised patients and found that they had a significantly higher risk of colonisation with MRSA. Screening of family members should therefore supplement staff screening. 
No benefits in any form have been received or will be received from a commercial party related directly or indirectly to the subject of this article.

\section{References}

1. Tiemersma EW, Bronzwaer SL, Lyytikainen 0, et al. Methicillin-resistant Staphylococcus aureus in Europe, 1999-2002. Emerg Infect Dis 2004;10:1627-34.

2. Boyce JM. Methicillin-resistant Staphylococcus aureus: a continuing infection control challenge. Eur J Clin Microbiol Infect Dis 1994;13:45-9.

3. Mitsuda T, Arai K, Ibe M, et al. The influence of methicillin-resistant Staphylococcus aureus (MRSA) carriers in a nursery and transmission of MRSA to their households. J Hosp Infect 1999; 42:45-51.

4. Cosgrove SE, Sakoulas G, Perencevich EN, et al. Comparison of mortality associated with methicillin-resistant and methicillin-susceptible Staphylococcus aureus bacteremia: a meta-analysis. Clin Infect Dis 2003;36:53-9.

5. Abramson MA, Sexton DJ. Nosocomial methicillin-resistant and methicillin-susceptible Staphylococcus aureus primary bacteremia: at what costs? Infect Control Hosp Epidemiol 1999;20:408-11.

6. Herr CE, Heckrodt TH, Hofmann FA, Schnettler R, Eikmann TF. Additional costs for preventing the spread of methicillin-resistant Staphylococcus aureus and a strategy for reducing these costs on a surgical ward. Infect Control Hosp Epidemiol 2003;24:673-8

7. Solberg CO. Spread of Staphylococcus aureus in hospitals: causes and prevention. Scand J Infect Dis 2000;32:587-95.

8. Blok HE, Troelstra A, Kamp-Hopmans TE, et al. Role of healthcare workers in outbreaks of methicillin-resistant Staphylococcus aureus: a 10-year evaluation from a Dutch university hospital. Infect Control Hosp Epidemio/ 2003;24:679-85.

9. Reagan DR, Doebbeling BN, Pfaller MA, et al. Elimination of coincident Staphylococcus aureus nasal and hand carriage with intranasal application of mupirocin calcium ointment. Ann Intern Med 1991;114:101-6.

10. Allen KD, Anson JJ, Parsons LA, Frost NG. Staff carriage of methicillin-resistant Staphylococcus aureus (EMRSA 15) and the home environment: a case report. J Hosp Infect 1997;35:307-11.
11. Masterton RG, Coia JE, Notman AW, Kempton-Smith L, Cookson BD. Refractory methicillin-resistant Staphylococcus aureus carriage associated with contamination of the home environment. J Hosp Infect 1995;29:318-19.

12. Muder RR, Brennen C, Goetz AM. Infection with methicillin-resistant Staphylococcus aureus among hospital employees. Infect Control Hosp Epidemio/ 1993;14:576-8.

13. Simmons BP, Munn C, Gelfand M. Toxic shock in a hospital employee due to methicillin-resistant Staphylococcus aureus. Infect Control 1986;7:350.

14. Cox RA, Conquest C. Strategies for the management of healthcare staff colonized with epidemic methicillin-resistant Staphylococcus aureus. J Hosp Infect 1997:35:117-27.

15. No authors listed. Dutch Working Party for Infection Prevention. MRSA Ziekenhuis. MRSA in nursing homes. 2005. available at www.WIP.nl (date last accessed 11 May 2007).

16. No authors listed. Empfehlung zur prävention und Kontrolle von methicillin-resistenten Staphylococcus aureus-Stämmen (MRSA) in Krankenhäusern under anderen medizinischen Einrichtungen. Mitteilung der Kommission für Krankenhaushygiene und Infektionsprävention am RKI. Bundesgesundheitbsbl-Gesundheitsforsch-Geseundheitsschutz 1999:42:954-6.

17. Garner JS. Guideline for isolation precautions in hospitals: The Hospital Infection Control Practices Advisory Committee. Infect Control Hosp Epidemiol 1996;17:53-80.

18. Bowler I. Strategies for the management of healthcare staff colonized with epidemic methicillin-resistant Staphylococcus aureus. J Hosp Infect 1997;36:321-2.

19. Lessing MP, Jordens JZ, Bowler IC. When should healthcare workers be screened for methicillin-resistant Staphylococcus aureus? J Hosp Infect 1996;34:20510.

20. Rohr U, Mueller C, Wilhelm M, Muhr G, Gatermann S. Methicillin-resistant Staphylococcus aureus whole-body decolonization among hospitalized patients with variable site colonization by using mupirocin in combination with octenidine dihydrochloride. J Hosp Infect 2003;54:305-9.

21. Eveillard M, Martin Y, Hidri N, Boussougant Y, Joly-Guillou ML. Carriage of methicillin-resistant Staphylococcus aureus among hospital employees: prevalence, duration, and transmission to households. Infect Control Hosp Epidemiol 2004;25:114-20.

22. Calfee DP, Durbin LJ, Germanson TP, et al. Spread of methicillin-resistant Staphylococcus aureus (MRSA) among household contacts of individuals with nosocomially acquired MRSA. Infect Control Hosp Epidemiol 2003;24:422-6. 